\title{
Modeling and Performance Analysis of Giant Magnetostrictive Microgripper with Flexure Hinge
}

\author{
Qinghua Cao*, Quanguo Lu, Junmei Xi, Jianwu Yan, and Changbao Chu \\ Institute of Micro/nano Actuation and Control, Nanchang Institute of Technology, \\ Nanchang, P.R. China \\ qh9863@126.com, luqg@whut. edu.cn
}

\begin{abstract}
In order to improve the steady output of micrgripper, the giant magnetostrictive micrgripper with flexure hinge is taken as the research object. Its structure and working principle are presented the static performance experiments are done and the better linear work domain is found. On the basis of that the dynamic model of the microgripper is established and the formula of the microgripper's output displacement is deduced. the relationship between the microgripper's parameters and responses are analyzed and open-loop simulation step is done, The simulation results show the giant magnetostrictive material(GMM) rod damp effects on the response time and its quality has little influence.
\end{abstract}

Keywords: Giant magnetostrictive micrgripper, Flexure hinge, Dynamic model, Open-loop simulation step.

\section{Introduction}

Microgripper[1] fulfils the core role in fine handling micro- parts, which can convert other power into mechanical energy. In recent years, the research of the micrgripper are concentrated in two respects with the rapid development of tiny technology : One is the drive mode, for example electrostatic microgripper, thermally driven microgripper [2], piezoelectric driven microgripper [3], shape memory alloy microgripper [4], and vacuum adsorption microgripper, etc. on the other hand, Many studies have been conducted to develop microgripper technology.

In this study, the giant magnetostrictive micrgripper with flexure hinge is taken as the research object. The static performance experiments are done and the better linear work domain is found. On the basis of that the dynamic model of the microgripper is established and open-loop simulation step is done.

\section{Structure and Static Performance Experiments}

\subsection{Structure Design of the Microgripper}

Magnetostrictive microgripper is a new microgripper[5], which is produced by GMM doing work through the stretching deformation of it in changed magnetic field. Fig. 1 shows a conceptual drawing of the microgripper proposed in this study.

\footnotetext{
* Corresponding author.
} 


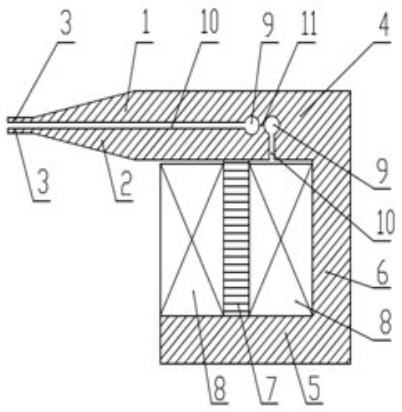

1. palate

2. microgripper jaw

3. JAW

4. uppersuspensionarm

5. suspensionarm

6. linker arm

7. GMM

8. Electromagnetic coil

9. Circular hole

10. Slice

11. Flexure hinge

Fig. 1. Conceptual drawing of microgripper

The principle of operation of the proposed microgripper is as follows: firstly, GMM is placed between the microgripper jaw and the suspensionarm, A magnetic circuit is formed through the microgripper jaw , uppersuspensionarm, linker arm, suspensionarm, GMM.When the controlled current is flowing into the electromagnetic coil to produce magnetic field, GMM produces stretching deformation. Secondly, Deformation can promote the microgripper jaw around the flexible hinge towards the direction of palate, so that the JAW produces microdisplacement to clamping objects.

\subsection{Static Performance Experiments}

the PC machine is adopted in the measurement and control system, digital signals which PC generate converted into analog voltage signals by PCI-8333D/A, which input to the controllable constant current source. the coil is driven from current source so that the magnetic field strength around the rod is altered, the GMM rod produces magnetostrictive deformation in order to provide microgripper driving, accordingly,displacement and clamping force of JAW is generated.Experimental system is placed on vibration isolation platform in order to reduce effect of the ground vibration. At the same time, environmental humidity and temperature stability are to meet the requirement of experiment. Experimental In-kind photo is shown in Fig 2.

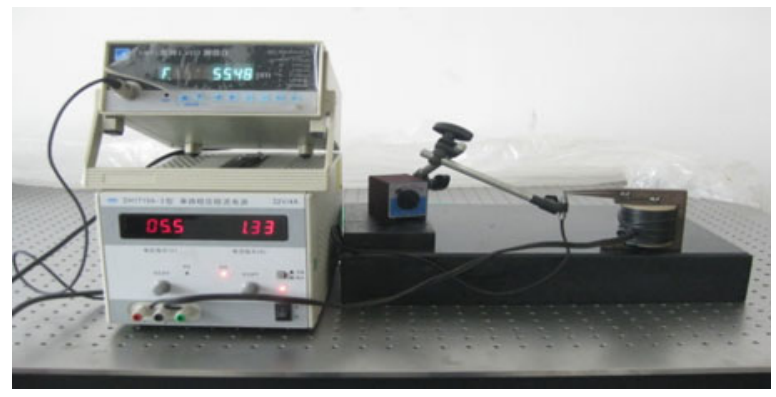

Fig. 2. Experimental In-kind photo

The controlled current began to increase from 0 to $4 \mathrm{~A}$, experimental curve is drawn, which are shows in Fig .3. There is the characteristic of large-time delay of the 
giant magnetostrictive material(GMM), the linear degrees are relatively poor, to simplify the mathematics model, the better linear work domain for $0.6 \sim 1$. 5A is found by the test and analysis.

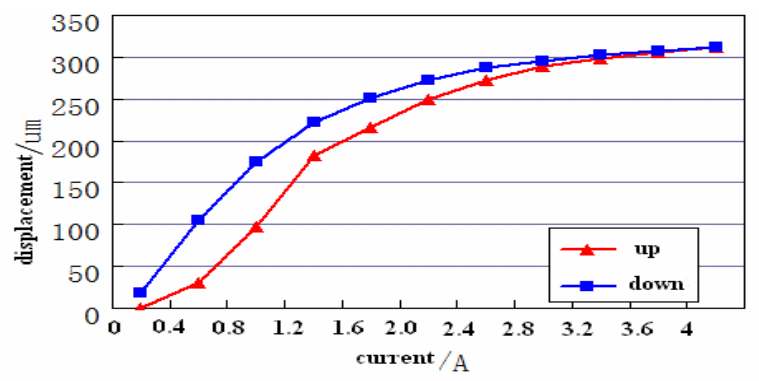

Fig. 3. Static performance experiments

\section{Displacement-Current Modle of the Microgripper}

\subsection{The Linear Model of GMM}

Under the environment of steady current, the GMM rod is elongated due to the magnetic field, which generate driving force $f_{q}$ and elastic restoring force $f_{h}$, Supposed the rod as an ideal elastomer, $\mathrm{x}$ is the rod elongation, according to Hooker's theorem, elastic restoring force function $\mathrm{f}_{\mathrm{h}}$ can be expressed as:

$$
f_{h}=K_{G M M} x
$$

Where $\mathrm{K}_{\mathrm{GMM}}$ is the rod stiffness coefficient, From mechanics of materials, function $\mathrm{K}_{\mathrm{GMM}}$ is defined as:

$$
K_{G M M}=\frac{E_{1} A}{l_{m}}
$$

Where $E_{1}$ is Young's modulus of the length direction, $A$ is cross-sectional area and $1_{m}$ is the rod length.

Neglecting the other force, force diagram is described in Fig. 4.

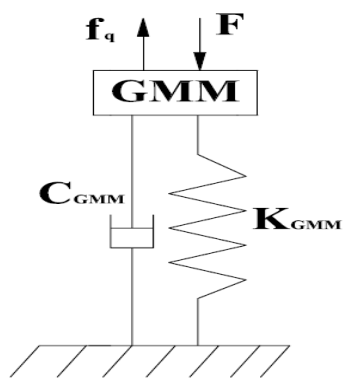

Fig. 4. Force diagram of GMM 
In the light of d'Alembert's principle, function F can be written as:

$$
F=f_{q}-K_{g m m} x-C_{g m m} \dot{x}-M_{g m m} \ddot{x}
$$

Where $\mathrm{F}$ is the force which the rod is received from the microgripper jaw.

Overlooking the temperature change, and only considering the axial deformation the length, literature describes the following linear piezomagnetic equation:

$$
\begin{aligned}
\varepsilon & =\frac{\sigma}{E_{1}}+d H \\
B & =d \sigma+\mu H
\end{aligned}
$$

Where $\varepsilon$ is the total axial strain, $\mathrm{d}$ is piezomagnetic coefficient , $\mu$ is magnetic permeability and $\mathrm{H}$ is Magnetic field strength.

Both sides of (4) are multiplied by the rod length $\mathrm{lm}$, the rod elongation function $\mathrm{x}$ can be expressed as:

$$
x=\frac{\sigma \cdot l_{m}}{E_{1}}+d \cdot H \cdot l_{m}
$$

According to Ampere's theorem, the magnetic field around rod is uniform magnetic field, and magnetic field strength at electromagnetic coil center is approximately equal to the average intensity of rod, one can get the following relations:

$$
N I=K_{\text {coil }} H l_{m}
$$

Where $\mathrm{K}_{\text {coil }}$ is the compensatory coefficient of the drived coil.

Substituting (7) into (6) and rearranging gives:

$$
x=\frac{\sigma \cdot l_{m}}{E_{1}}+\frac{d \cdot N I}{K_{c o i l}}
$$

Substituting (1),(2),(6),(8)into(3), function F can be rewritten as follows:

$$
F=\frac{E_{1} A d N}{K_{\text {coil }} l_{m}} i-K_{g m m} x-C_{g m m} \dot{x}-M_{g m m} \ddot{x}
$$

Where reference [6] is cited, driving force function $\mathrm{f}_{\mathrm{q}}$ is implemented as:

$$
f_{q}=\frac{E_{1} \cdot A \cdot d \cdot N}{l_{m}} I
$$

\subsection{The Design of Flexure Hinge}

The structure of Flexible hinge is shown in Figure 5, where $t$ is the minimum thickness of flexible hinge, $a=t+2 R-2 R \cos \theta$ is infinitesimal thickness, $d u=d(R \sin \theta)$ is the infinitesimal length, around the $\mathrm{z}$-axis the moment of inertia function[7] $\mathrm{Iz}=\mathrm{ba} 3 / 12$. 


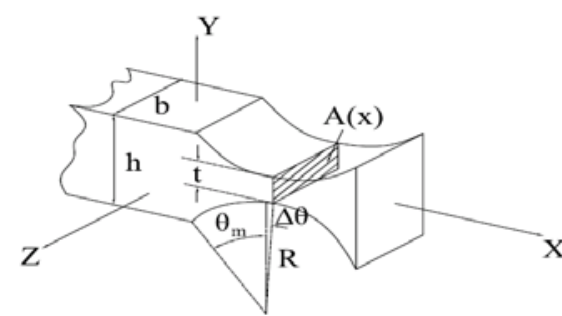

(a) Structure diagram

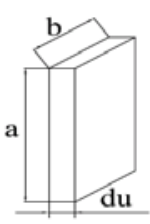

(b) Infinitesimal geometry

Fig. 5. Analysis of flexible hinge

Supposing deformation of flexible hinge isr pure bending, ignoring angular displacement except the flexible hinge, $\mathrm{E}_{2}$ is Young's modulus of the microgripper, the deflection angle function as follows:

$$
\alpha_{z}=\int_{z} \frac{M_{z}(x) d x}{E_{2} I_{z}(x)}=\int_{-\theta_{m}}^{\theta_{m}} \frac{12 M_{z} R \cos \theta}{E_{2} b(t+2 R-2 R \cos \theta)^{3}} d \theta
$$

Here $s=R / t$, function $\alpha_{z}$ can be rewritten as:

$$
\begin{aligned}
& \alpha_{z}=\frac{12 M_{z}}{E_{2} b R^{2}} \int_{-\theta_{m}}^{\theta_{m}} \frac{\cos \theta}{(t+2 R-2 R \cos \theta)^{3}} d \theta \\
& =\frac{12 M_{z}}{E_{2} b R^{2}} \int_{-\theta_{m}}^{\theta_{m}} \frac{\cos \theta}{(s+2-2 \cos \theta)^{3}} d \theta
\end{aligned}
$$

Torsional stiffness of flexible hinge function $\mathrm{k}$ can be derived as follows:

$$
k=\frac{M_{z}}{\alpha_{z}}=\frac{E_{2} b R^{2}}{12 \int_{-\theta_{m}}^{\theta_{m}} \frac{\cos \theta}{(s+2-2 \cos \theta)^{3}} d \theta}=\frac{E_{2} b R^{2}}{12 f}
$$

Where

$$
\begin{aligned}
& f=\int_{-\theta_{m}}^{\theta_{m}} \frac{\cos \theta}{(s+2-2 \cos \theta)^{3}} d \theta \\
& =\frac{8 s^{4}(2 s+1) \tan \frac{\theta_{m}}{2}}{(4 s+1)^{2}\left[1+(4 s+1) \tan ^{2} \frac{\theta_{m}}{2}\right]^{2}} \\
& +\frac{4 s^{3}\left(6 s^{2}+3 s+1\right) \tan \frac{\theta_{m}}{2}}{(4 s+1)^{2}\left[1+(4 s+1) \tan { }^{2} \frac{\theta_{m}}{2}\right]} \\
& +\frac{12 s^{4}(2 s+1)}{(4 s+1)^{5 / 2}} \arctan \left(\sqrt{(4 s+1)} \tan \frac{\theta_{m}}{2}\right)
\end{aligned}
$$


According to principles of Action and Reaction, force of the microgripper received from GMM is equal to the force of microgripper jaw acting on the GMM, F is external force of the flexible hinge, Leverage model simplified from flexible hinge is shown in Fig.6.

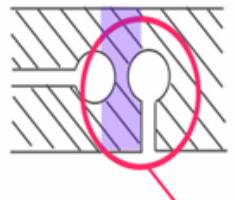

\section{flexure hinge}

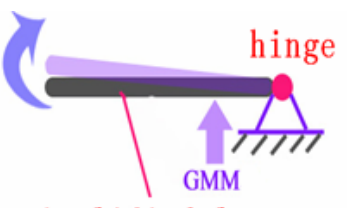

simplified leverage

(b)

Fig. 6. Leverage model (b) simplified from flexible hinge (a)

Because the rod elongation $\mathrm{x}$ is very small, the angle of leverage rotation $\theta=\frac{x}{m}$, where $\mathrm{m}$ is distance from the rod to the hinge simplied. Stored elastic energy $A_{\theta}$ of the flexible hinge can be expressed as: $A_{\theta}=\frac{1}{2} k \theta^{2}$, elastic energy $A_{\theta}$ is equal to work done from force $\mathrm{F}$, work function $\mathrm{A}$ can be defined as: $A=f x$, force $\mathrm{F}$ can be rewritten again:

$$
F=\frac{k x}{m^{2}}
$$

Substituting (10),(11) into (9) and rearranging gives:

$$
M_{g m m} \ddot{x}+C_{g m m} \dot{x}+\left(K_{g m m}+\frac{K}{m^{2}}\right) x=\frac{E_{1} A d N}{K_{c o i l} l_{m}} i
$$

\subsection{Displacement of JAW}

According to leverage shown in Fig.4, one can get the following relations:

$$
\frac{y}{h}=\frac{x}{m}
$$

Where $\mathrm{h}$ is the distance from JAW to the hinge, $\mathrm{y}$ is the displacement of JAW.

Substituting (12) into (14) and rearranging gives:

$$
\frac{m}{h} M_{g m m} y+\frac{m}{h} C_{g m m} y+\frac{m}{h}\left(K_{g m m}+\frac{K}{m^{2}}\right) y=\frac{E_{1} A d N}{K_{\text {coil }} l_{m}} i
$$




\section{Experiments of the Microgripper}

Laplace transform of (14) is done and rearranging gives:

$$
G(s)=\frac{x(s)}{I(s)}=\frac{\frac{E_{1} A d N}{K_{c o i} l_{m}}}{\frac{m}{h} M_{g m m} s^{2}+\frac{m}{h} C_{g m m} s+\frac{m}{h}\left(K_{g m m}+\frac{K}{m^{2}}\right)}
$$

The relevant parameters of (15) is given and function $G(s)$ can be rewritten as:

$$
\begin{aligned}
& G(s)=K^{*} \frac{\omega_{n}^{* 2}}{s^{2}+2 \xi^{*} \omega_{n}^{*} s+\omega_{n}^{* 2}} \\
& =2.71 \times 10^{-4} \times \frac{519700^{2}}{s^{2}+2 \times 0.39 \times 51970 s+51970^{2}}
\end{aligned}
$$

Where $\mathrm{K}^{*}$ is open-loop gain, $\xi^{*}$ is the damp coefficient, $\omega_{n}^{*}$ is angular frequency of the undamped oscillation.

The relationship between the microgripper's parameters and responses are analyzed and open-loop simulation step is done, steps are as follows: firstly, the GMM damp is changed when the other parameters remain, the simulation result is shown in Fig.7. secondly, the GMM quality is changed when the other parameters remain, the simulation result is shown in Fig.8.

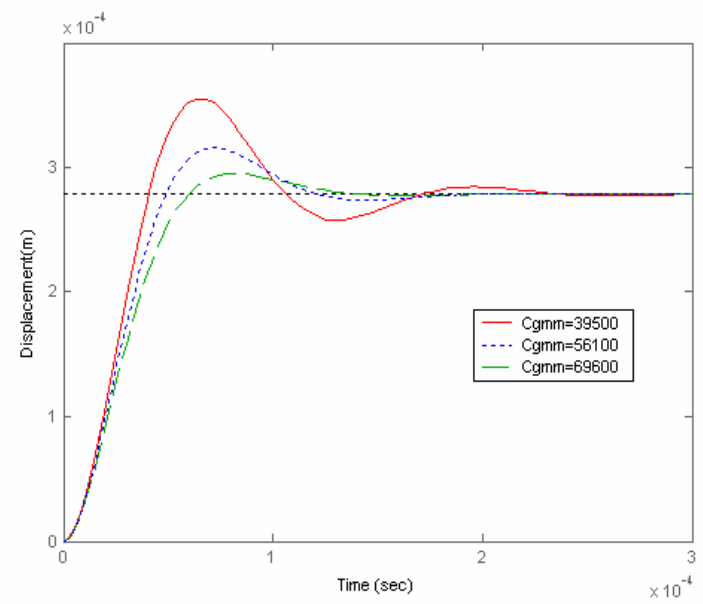

Fig. 7. Comparison of the simulation curves with the different damp 


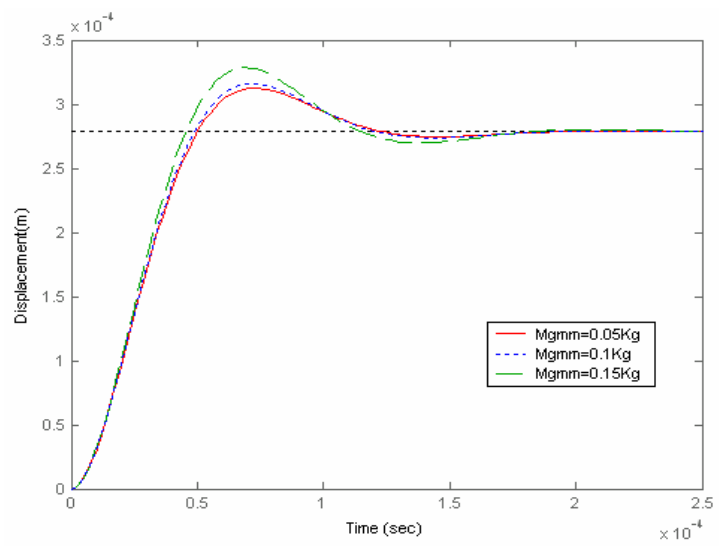

Fig. 8. Comparison of the simulation curves with the different quality

The simulation results show the giant magnetostrictive material(GMM) rod damp effects on the response time and its quality has little influence, At the same time, the overshoot decrease due to the damp increase, while the overshoot iecrease due to the quality increase. Accordingly, the relationship between the microgripper's designed parameters and responses are found, It is beneficial for the microgripper to optimize parameter.

\section{Conclusion}

In this study, the giant magnetostrictive micrgripper with flexure hinge is taken as the research object. Firstly, due to the hysteresis and nonlinearity of GMM, the better linear work domain is found when the static performance experiments are finished. Secondly, a flexible hinge mechanism to magnify the displacement is prototyped and torsional stiffness are analyzed, with compact structure, small size, motion sensitivity. the dynamic model of the microgripper is established and the formula of the microgripper's output displacement is deduced. Lastly, open-loop simulation step is finished, The simulation results show the giant magnetostrictive material(GMM) rod damp effects on the response time and its quality has little influence . there lie foundation for follow-up parameter optimized research.

\section{Acknowledgment}

The authors would like to acknowledge the financial support by the National Natural Science Fund of China (Grant No. 50865008), China Postdoctoral Science Fund (Grant No. 20080431005) and Youth Science Fund of Jiangxi province office of education of China (Grant No.GJJ09611, Grant No. GJJ10633). 


\section{References}

[1] Anis, Y.H., Mills, J.K., Cleghorn, W.L.: Active microgripper interface used in microassembly of MEMS. In: Canadian Conference on Electrical and Computer Engineering, CCECE 2006, May 2006, pp. 352-354 (2006)

[2] Elbuken, C., Lin, G., Ren, C.L., Yavuz, M., Khamesee, M.B.: A monolithic polymeric microgripper with photo-thermal actuation for biomanipulation. In: IEEE International Conference on Mechatronics and Automation, ICMA 2008, pp. 707-711.

[3] Nah, S.K., Zhong, Z.W.: A microgripper using piezoelectric actuation for micro-object manipulation. Sensors and Actuators: A 133, 218-224 (2007)

[4] Chang, R.J., Lin, Y.C., Shiu, C.C., Hsieh, Y.T.: Development of SMA-actuated microgripper in micro assembly applications. In: 33rd Annual Conference of the IEEE Industrial Electronics Society, IECON 2007, pp. 2886-2891 (2007)

[5] Jia, Z., Guo, D.: Theory and Applications of Giant Magnetostrictive Microdisplacement Actuator. Science and Technology Press, Beijing (2008)

[6] Cao, Q., Lu, Q., Yan, J., Liu, D.: Design of New Microgripper Based on GMM. In: The 1st International Conference on Mechanic Automation and Control Engineering, June 25-27 (2010) 